\title{
Patología mamaria benigna como hallazgo en mastoplastia reductiva
}

\section{Benign mammary pathology as a finding in reductive mastoplasty}

\author{
Ximena Zozaya Minichini, * Lucía Escobedo Berumen, ${ }^{\neq}$ \\ Karen Moedano Rico, ${ }^{\circledR}$ Ricardo Balanzá López, "l Manuel García Velasco"
}

\section{Resumen}

Objetivo: Valorar la incidencia de la patología mamaria benigna (PMB) en las piezas patológicas de pacientes sometidas a mastoplastia reductiva. Se estudiaron los reportes de patología de toda paciente sometida a mastoplastia reductiva (MR) en un periodo de 10 años. Se buscó la asociación entre ciertas variantes y la presencia de patología mamaria benigna. De 314 pacientes, $136(43.3 \%)$ presentaron patología mamaria benigna. La lesión más común fue mastopatía fibroquística $(47.7 \%, n=65)$, que representó el $60.3 \%(n=41, p=0.004)$ de las lesiones en mayores de 40 años. La segunda lesión más común fue la hiperplasia ductal sin atipia $(46.3 \%, n=63)$, que predominó en menores de 40 años. Los fibroadenomas fueron las lesiones más comunes en menores de 40 años ( $p$ $=0.002)$. Se encontró el 82.35\% $(n=14, p=0.004)$ de las microcalcificaciones en las pacientes mayores de 40 años. Conclusiones: Encontramos una relación directa entre la edad y el tipo de lesión. La incidencia de patología mamaria benigna tras mastoplastia reductiva en este estudio fue del $43.3 \%$, porcentaje por debajo del $76.2 \%$ reportado en la literatura. Este estudio nos ayuda a aumentar la consciencia del equipo médico sobre la posible presencia de lesiones en la pieza patológica de cirugía mamaria electiva en pacientes sanas.

Palabras clave: Patología mamaria benigna, mastoplastia reductiva.
* Cirujana de Mama del Hospital Ángeles Lomas, Estado de México.

₹ Pediatra del Instituto Nacional de Pediatría, Ciudad de México.

$\S$ Residente de tercer año de Cirugía General, Hospital Ángeles Lomas, Estado de México.

॥ Cirujano, Residente de primer año de Endoscopia, Hospital Médica Sur, Ciudad de México.

^ Cirugía Plástica y Reconstructiva. Jefe de Educación Médica del Hospital Ángeles Lomas, Estado de México.

Correspondencia:

Dr. Ximena Zozaya Minichini

Correo electrónico: ximezm@gmail.com

Aceptado: 26-09-2019.

www.medigraphic.com/actamedica
Abstract

Objective: To assess the incidence of benign breast disease (BBD) in pathological specimens of patients undergoing reductive mastoplasty (RM). We studied the pathology reports of patients undergoing RM in a period of 10 years. The association between certain variants and the presence of BBD was analyzed. Of the total 314 patients, 136 (43.3\%) presented BBD. The most common lesion was fibrocystic mastopathy $(47.7 \% n=65)$, representing $60.3 \%(n=41 p=0.004)$ of lesions in patients older than 40 years. The second most common lesion was ductal hyperplasia without atypia $(46.3 \% n=63)$, predominating in those under 40 years. Fibroadenomas were the most common lesions in those under 40 years $(p=0.002)$. $82.35 \%(n=14 p=0.004)$ of microcalcifications were in women older than 40 years. Conclusions: We found a relationship between age and type of injury. The incidence of BBD after RM in this study was $43.3 \%$, percentage below $76.2 \%$ reported in the literature. This study helps us increase awareness in the medical community about the possible presence of lesions in the pathological specimen after elective breast surgery in healthy patients.

Keywords: Benign mammary pathology, reductive mastoplasty.

\section{INTRODUCCIÓN}

La macromastia, también conocida como gigantomastia o hipertrofia mamaria, es una situación benigna que implica la hipertrofia mamaria y resulta en dolor cervical, dorsal y de hombros, cifosis, excoriaciones de los tirantes del sostén, intertrigo crónico en el pliegue inframamario, limitación en el ejercicio y en las actividades diarias y, en la mayoría de los casos, problemas de autoestima. La mastoplastia de reducción (MR) ha demostrado ser efectiva en el alivio de todas estas situaciones. ${ }^{1}$ La MR es uno de los procedimientos quirúrgicos más comúnmente practicados en cirugía plástica. $^{2-4}$ En EUA se calculan 110,000 procedimientos de MR/año. ${ }^{1}$ La mayoría de los procedimientos bilaterales 
se realizan por macromastia sintomática, pero de forma unilateral se realiza para corregir la asimetría en pacientes con diferencias congénitas o que fueron sometidas a cirugía por cáncer de mama. ${ }^{3,5}$

Se calcula que un 30 a $60 \%$ de las MR se realiza en mujeres mayores de 40 años, edad en la que aumenta el riesgo de cáncer de mama. La incidencia es más grande en mujeres mayores de 50 años, especialmente si el procedimiento se realiza con propósitos de simetría en pacientes con antecedente de cáncer de mama contralateral. ${ }^{6}$ Dado que el riesgo de vida de cualquier mujer de padecer cáncer de mama es de una en cada ocho mujeres, no es infrecuente encontrar lesiones cancerosas o precancerosas en los especímenes de MR. ${ }^{5}$ En el estudio de Tadler y colaboradores, ${ }^{2}$ se detectaron lesiones en $76.2 \%$ de los especímenes estudiados; de éstos, el $72.5 \%$ fueron lesiones benignas no proliferativas y proliferativas sin atipia, el $2.8 \%$ se constituyó por lesiones proliferativas con atipia y $0.9 \%$ por carcinoma in situ. Dos terceras partes de las lesiones malignas halladas fueron no invasivas y ocultas por mastografía, las cuales fueron encontradas únicamente por muestreo a ciegas de la pieza enviada a patología.

El manejo en estas situaciones se complica por las siguientes dos razones: 1) dada la naturaleza del procedimiento quirúrgico, la muestra generalmente llega al patólogo en trozos. 2) La mayoría de las veces es imposible orientarla o determinar el tamaño de la lesión y sus márgenes. ${ }^{6}$ Gracias a la imposibilidad de determinar los bordes, el tamaño de la lesión y el estado de los márgenes, la mastectomía ha sido el procedimiento de elección en el cáncer diagnosticado como hallazgo tras MR.

El propósito de este estudio es el de valorar la incidencia de los diferentes tipos de lesiones mamarias benignas en pacientes sometidas a mastoplastia reductiva, en un periodo de 10 años, tomando en consideración el tipo de lesión, la presencia de estudios de imagen previos al procedimiento y los antecedentes de cáncer de mama de las pacientes, con el fin de determinar la incidencia de lesiones mamarias benignas en la población estudiada, la relación entre los hallazgos histopatológicos y las variables estudiadas; también se buscó analizar nuestro método de procesamiento de las piezas patológicas.

\section{MATERIAL Y MÉTODOS}

Se estudiaron los reportes de patología de toda paciente sometida a mastoplastia reductiva de un cirujano plástico solamente en el Hospital Ángeles Lomas, durante el periodo comprendido entre enero del 2006 a diciembre del 2016.

Del total de reportes de patología analizados $(n=$ 351), se descartaron 10 pacientes por pertenecer al sexo masculino y 27 pacientes porque el procedimiento final consistió únicamente en resección de nódulos benignos, sin involucrar una mastoplastia reductiva en sí. La muestra final resultó ser de 314 pacientes. De este total $(n=314)$, el $43.3 \%(n=136)$ presentó reporte de positivo para patología mamaria benigna.

Los criterios de inclusión fueron los siguientes: sexo femenino, de cualquier edad y sometidas a mastoplastia reductiva en el periodo de tiempo mencionado. Los criterios de exclusión fueron: pacientes del sexo masculino y aquellas pacientes sometidas a resección de tejido mamario sin mastoplastia reductiva.

Toda paciente igual o mayor de 40 años contó con mastografía en el último año. Se realizó un ultrasonido mamario a toda paciente menor de 40 años con factores de riesgo para cáncer de mama o lesiones sospechosas al momento de la exploración física. Ninguna de las pacientes estudiadas presentó lesiones sospechosas en los estudios de imagen.

Se dividió a las pacientes con reporte de patología positivo para lesiones benignas $(n=136)$ en dos grupos: pacientes mayores de 40 años y menores de 40 años. En cada una de las pacientes se estudió el lado que contenía la lesión, edad, peso, uso de terapia hormonal, antecedente de paridad, si la cirugía fue realizada pre o postgesta, si las pacientes eran pre o postmenopáusicas y si la paciente presentaba antecedentes personales o heredofamiliares de cáncer de mama. Posteriormente se analizó la incidencia de cada patología específica y se comparó con algunas de las variables mencionadas.

En aquellas pacientes con múltiples diagnósticos histológicos se tomó el diagnóstico con peor pronóstico para el estudio. ${ }^{3,5}$

En nuestro Departamento de Patología, todos los especímenes de MR primero se analizan macroscópicamente. En ausencia de lesiones sospechosas y de factores de riesgo, se tomaron dos muestras al azar para el análisis histológico. En caso de encontrar lesiones sospechosas, se centró la atención en ellas y se tomó el número de muestras necesarias para su estudio integral. En pacientes mayores de 40 años y en aquéllas con factores de riesgo, el estudio se realizó con mayor cautela.

\section{RESULTADOS}

En cuanto al resultado de patología de la muestra total $(n=314), 136$ pacientes (43.3\%) presentaron hallazgos de patología mamaria benigna y 178 pacientes (56.6\%) tuvieron resultados negativos. La edad promedio de las pacientes con hallazgos de benignidad fue de 39 años, la edad mínima de 13 años y la máxima de 66 años de edad. Considerando el número de diagnósticos histopatológicos por paciente, el $64.4 \%(n=88)$ presentó más de un diag- 
nóstico, mientras que en el 31.6\% $(n=43)$ se realizó un solo diagnóstico.

En cuanto a la incidencia de patología mamaria benigna (Tabla 1), la patología más comúnmente encontrada fue hiperplasia ductal sin atipia, la cual representó el 46.3\% (n $=63$ ) de los reportes de patología positivos para patología mamaria benigna. La segunda patología más común fue la presencia de fibroadenomas, que fueron hallados en el $16.9 \%(n=23)$. Únicamente se encontró un caso de hiperplasia ductal con atipia $(0.7 \%)$ en una paciente con antecedente familiar de cáncer de mama.

En cuanto a la incidencia de PMB por edad (Tabla 2), los hallazgos positivos se encontraron en idéntica proporción entre pacientes menores y mayores de 40 años (50\%). Una vez más se tomó el diagnóstico de mayor riesgo en pacientes con hallazgos múltiples. En ambos grupos de edad, la lesión predominante fue la hiperplasia ductal sin atipia. En el grupo de pacientes mayores de 40 años, la segunda lesión más común fueron las microcalcificaciones, que representaron el $20.4 \%(n=14)$; dos de estas pacientes tuvieron antecedente familiar de cáncer de mama y una tercera fue sometida a mastoplastia de simetría por antecedente de cáncer de mama ipsilateral. Esta paciente además presentó hiperplasia lobulillar sin atipia.

Cuando analizamos todos los diagnósticos sin categorizarlos según el riesgo de malignización, encontramos que la lesión más común fue la enfermedad fibroquística $(47.7 \%, n=65)$, lo que representa el $60.3 \%(n=41, p$

Tabla 1: Incidencia de hallazgos de patología mamaria benigna $(n=136)$.

Lesión $\mathrm{n}(\%)$

$\begin{array}{lc}\text { Lesiones no proliferativas } & \\ \text { Fibroadenomas } & 23(16.9) \\ \text { Mastopatía fibroquística } & 13(9.6) \\ \text { Quistes } & 1(0.7) \\ \text { Metaplasia apocrina } & 13(9.6) \\ \text { Microfibromatosis } & 1(0.7) \\ \text { Lesiones proliferativas sin atipia } & \\ \text { Hiperplasia ductal sin atipia } & 63(46.3) \\ \text { Adenosis esclerosante } & 2(1.5) \\ \text { Microcalcificaciones } & 17(12.5) \\ \text { Hiperplasia lobulillar sin atipia } & 1(0.7) \\ \text { Papiloma intraductal } & 1(0.7) \\ \text { Lesiones proliferativas con atipia } & \\ \text { Hiperplasia ductal con atipia } & 1(0.7) \\ \text { Hiperplasia lobulillar con atipia } & 0(0)\end{array}$

Se tomó el diagnóstico con mayor riesgo en pacientes con múltiples diagnósticos.

\begin{tabular}{|c|c|c|}
\hline \multicolumn{3}{|c|}{$\begin{array}{l}\text { Tabla 2: Incidencia de hallazgos de } \\
\text { patología mamaria benigna por edad. }\end{array}$} \\
\hline Lesiones & $\begin{array}{c}<40 \text { años } \\
(n=68) \\
n(\%)\end{array}$ & $\begin{array}{c}>40 \text { años } \\
(n=68) \\
n(\%)\end{array}$ \\
\hline \multicolumn{3}{|l|}{ Lesiones no proliferativas } \\
\hline Fibroadenomas & $18(26.5)$ & $5(7.4)$ \\
\hline Mastopatía fibroquística & $6(8.8)$ & $7(10.3)$ \\
\hline Quistes & $1(1.5)$ & $0(0)$ \\
\hline Metaplasia apocrina & $9(13.2)$ & $4(5.9)$ \\
\hline Microfibromatosis & $0(0)$ & $1(1.5)$ \\
\hline \multicolumn{3}{|l|}{ Lesiones proliferativas sin atipia } \\
\hline Hiperplasia ductal sin atipia & $30(44.1)$ & $33(48.5)$ \\
\hline Adenosis esclerosante & $1(1.5)$ & $1(1.5)$ \\
\hline Microcalcificaciones & $3(4.4)$ & $14(20.4)$ \\
\hline Hiperplasia lobulillar sin atipia & $0(0)$ & $1(1.5)$ \\
\hline Papiloma intraductal & $0(0)$ & $1(1.5)$ \\
\hline \multicolumn{3}{|l|}{ Lesiones proliferativas con atipia } \\
\hline Hiperplasia ductal con atipia & $0(0)$ & $1(1.5)$ \\
\hline Hiperplasia lobulillar con atipia & $0(0)$ & $0(0)$ \\
\hline
\end{tabular}

$=0.004)$ de las pacientes mayores de 40 años y el $35.3 \%$ $(\mathrm{n}=24)$ de aquéllas menores de 40 años. En cuanto al lado de lesiones de mastopatía fibroquística, el $67.7 \%$ fue bilateral $(n=44)$, el $23.1 \%(n=15)$ derecho y el $9.2 \%(n$ $=6$ ) izquierdo. En cuanto a la paridad, se encontró una relación directa entre el antecedente gestacional positivo y la mastopatía fibroquística $(p=0.018)$.

Al analizar la incidencia global de las lesiones hiperplásicas, la hiperplasia ductal sin atipia fue la más frecuentemente encontrada $(98.43 \%, \mathrm{n}=63$ ) y el diagnóstico principal cuando se categorizó por riesgo de malignización en pacientes con múltiples diagnósticos histopatológicos $(47.05 \% \mathrm{n}=64)$. Únicamente se encontró un caso $(1.56 \%)$ de hiperplasia ductal con atipia en una paciente con antecedente familiar de cáncer de mama.

En cuanto a la edad, se encontró una relación con significancia estadística en cuanto a la presencia de enfermedad no proliferativa y proliferativa sin atipia, con una $p=0.003$. Igualmente se encontró relación entre aquellas pacientes con enfermedad proliferativa con atipia y edad mayor a 40 años ( $p$ $=0.008$ ). En cuanto a la historia gestacional, se encontró una relación entre paridad y el tipo de hiperplasia; la más común fue la hiperplasia no proliferativa en nuligestas $(p=0.023)$.

Los fibroadenomas se encontraron en $26.4 \%(n=36)$ de las pacientes, el $38.2 \%(n=26)$ se encontró en menores de 40 años y el $14.7 \%(n=10)$ en mayores de 40 años. En 
cuanto al lado de la lesión, el $47.2 \%(n=17)$ de éstas fue bilateral, el $30.6 \%(n=11)$ en el lado derecho y el $22.2 \%$ $(n=8)$ en el izquierdo. El $25 \%(n=9)$ de las pacientes con este hallazgo utilizaba terapia de reemplazo hormonal, lo que resulta en una asociación estadísticamente significativa a favor del uso de terapia hormonal y la presencia de fibroadenomas $(p=0.094)$.

Se encontraron microcalcificaciones en $12.5 \%$ de las muestras analizadas $(n=17)$. De éstas, cuatro pacientes (23.5\%) tuvieron antecedente de cáncer de mama, y una de ellas fue sometida a mastoplastia de simetría por antecedente de cáncer de mama ipsilateral; las otras tres tuvieron algún antecedente familiar. Ninguna de ellas presentó lesiones sospechosas en el análisis histopatológico del resto del tejido analizado. En las pacientes menores de 40 años, las microcalcificaciones solamente representaron el $17.6 \%(n=3)$ mientras que en mujeres mayores de 40 años representaron el $82.35 \%(n=14, p=0.004)$.

\section{DISCUSIÓN}

Petit y su equipo demostraron que el $1.6 \%$ de las pacientes sometidas a mastoplastia por simetría tras mastectomía por cáncer de mama contralateral presentó cáncer invasor al momento de la mastoplastia reductiva, ${ }^{7}$ por lo que se sugiere un análisis más detallado en los especímenes de estas pacientes. Gracias a estos hallazgos, nos podríamos preguntar si la mastoplastia reductiva reseca suficiente tejido mamario para disminuir el riesgo de cáncer de mama. Cuatro estudios grandes sugieren que la MR resulta en una reducción del riesgo relativo de $0.6 \%$ para padecer cáncer de mama, en comparación con la incidencia de cáncer de mama en la población general. ${ }^{7-10}$

Dupont y Page dividieron las lesiones mamarias benignas en tres categorías, dependiendo del riesgo de malignidad de cada una:

1. Lesiones no proliferativas: (RR: 1) quistes mamarios, cambios fibroquísticos, cambios apocrinos papilares, calcificaciones epiteliales, hiperplasia leve del tipo usual y metaplasia apocrina.

2. Lesiones proliferativas sin atipia: (RR: 1.3-1.9) hiperplasia ductal sin atipia, hiperplasia lobulillar sin atipia, papilomas intraductales, adenosis esclerosante, cicatrices radiales y fibroadenomas.

3. Lesiones proliferativas con atipia: (RR: 3.9-13) hiperplasia ductal atípica e hiperplasia lobulillar atípica. ${ }^{11}$ Tanto la enfermedad fibroquística como la hiperplasia ductal sin atipia son hallazgos comunes en MR.

Estudios previos han confirmado que las pacientes con lesiones proliferativas, hiperplasia ductal atípica e hiper- plasia lobulillar atípica tienen mayor riesgo de desarrollar cáncer de mama, junto con un riesgo absoluto de lesiones con atipia en un $30 \%$ a 25 años de seguimiento. ${ }^{12}$ La identificación de estas lesiones puede ayudar a reconocer a aquellas mujeres con riesgo de desarrollar cáncer de mama. ${ }^{1}$ Según un artículo publicado en 2016 por Päivi y otros, las lesiones proliferativas sin atipia aumentan el riesgo de cáncer de mama de 1.5-2 veces, la hiperplasia ductal y lobulillar con atipia lo aumenta 4-5 veces y el carcinoma lobulillar in situ (CLIS) aumenta el riesgo de padecer cáncer de mama de $8-10$ veces. ${ }^{4}$

El riesgo de encontrar lesiones de alto riesgo (hiperplasia ductal con atipia o carcinoma ductal o lobulillar in situ) en especímenes de MR para macromastia sintomática varía del $0.6-4.6 \% .^{1-3,6,13-16}$ Tadler y su equipo ${ }^{2}$ encontraron que la prevalencia de carcinoma in situ incidental en especímenes de mastoplastia reductiva fue significativamente mayor en pacientes con historia previa de cáncer contralateral que en aquellas pacientes sin historia previa de cáncer ( $5.5 \%$ versus $0.4 \%$ ). Este porcentaje varía de un estudio a otro gracias a la falta de uniformidad en el método de procesamiento de las muestras patológicas, ya que no existe un procedimiento mundialmente estandarizado que unifique la forma de hacerlo. ${ }^{3}$ Lógicamente, entre mayor tejido se estudie, mayor riesgo de encontrar lesiones premalignas o malignas. La incidencia de hallazgos de patología mamaria benigna tras este tipo de intervenciones ha sido menos estudiada. ${ }^{1}$

$\mathrm{Al}$ analizar el espécimen en el departamento de patología, inicialmente se requiere una valoración macroscópica detallada para identificar zonas sospechosas. ${ }^{17}$ Tras la valoración macroscópica, cada centro determina la forma del estudio histológico en bloques. Las piezas de las MR generalmente son grandes y la selección de pocas muestras representa un porcentaje pequeño del tejido enviado. ${ }^{18}$

Muchos estudios han abordado este mismo dilema: ¿cuántos bloques se recomienda estudiar para obtener una muestra fidedigna del tejido? ${ }^{1,3,5,18-21}$ Un estudio concluyó que, en pacientes menores de 30 años, se requiere un análisis macroscópico detallado, además de un estudio microscópico de al menos dos bloques. En pacientes mayores de 40 años se requiere una valoración microscópica más detallada, aun en ausencia de lesiones macroscópicas. ${ }^{22} \mathrm{Sin}$ embargo, Abiy y su equipo concluyeron que un aumento en el número de muestras analizadas se asocia directamente con una mayor frecuencia de hallazgos patológicos. En este estudio, con el análisis de tres muestras, los hallazgos fueron del $40 \%$, mientras que, al aumentar el número de muestras a ocho, este porcentaje se duplicó a 80\%. Un aumento en el número de muestras únicamente fue significativo para hallazgos patológicos en mujeres mayores de 40 años. Ninguna de las pacientes con hallazgos patológicos mostró lesiones en las mastografías previas a la cirugía. ${ }^{3}$ Nielsen y 
los otros realizaron un análisis patológico en 83 autopsias de mujeres jóvenes, y encontraron (con estudios extensos del tejido) que el $2 \%$ de las mujeres tenía cáncer de mama invasivo oculto, el 14\% carcinoma ductal in situ (CDIS), $3 \%$ carcinoma lobulillar in situ (CLIS) y $7 \%$ hiperplasia ductal atípica. Estos resultados soportan la hipótesis de que un mayor número de muestras conlleva a un mayor número de hallazgos de lesiones significativas. ${ }^{15,22}$ Dotto y colaboradores realizaron un estudio de los hallazgos histológicos de mastoplastias reductivas de 145 pacientes. En 35 casos (45.5\%) se obtuvieron resultados normales, en $30(38.9 \%)$ se obtuvieron resultados de patología benigna y en 12 (15.6\%) se obtuvieron lesiones limítrofes. No hubo hallazgos de patología maligna en este estudio. ${ }^{15}$

Un estudio realizado por Bondeson y colaboradores ${ }^{23}$ concluyó que, en pacientes menores de 30 años, un análisis macroscópico detallado con uno o dos bloques para el análisis microscópico era suficiente. Sin embargo, para mujeres mayores de 40 años, se recomienda un estudio microscópico extenso aún en ausencia de anormalidades macroscópicas. En este estudio, determinaron que, para MR de rutina, cuatro bloques para el estudio microscópico eran suficientes, incluyendo tejido mamario y una muestra de piel. También, con este método, determinaron la presencia de carcinoma en $0.2 \%$ de las pacientes. $\mathrm{Al}$ aumentar el número de muestras, incrementa la posibilidad de encontrar lesiones, especialmente en mujeres mayores de 50 años.

En su estudio, Tadler y su equipo ${ }^{2}$ analizaron las piezas patológicas de MR de 534 pacientes de 1990 a 2010. En el examen histopatológico, se examinaron secciones de 1 $\mathrm{cm}$ de espesor y se tomaron de 2-4 muestras de tejido al azar de cada mama y se cortaron en espesor de 4-5 mm. Cuando se detectó cáncer de mama microscópicamente, se tomaron muestras adicionales y se valoraron los márgenes. En este estudio, se detectaron lesiones en $76.2 \%$ de las pacientes, $72.5 \%$ de las cuales eran lesiones no proliferativas y proliferativas sin atipia, $2.8 \%$ proliferativas con atipia y carcinoma in situ en el $0.9 \%$. Ambaye y su grupo ${ }^{3}$ estudiaron 202 casos de mastoplastia reductiva entre junio 2006 a agosto 2007; concluyeron que, con el análisis de tres muestras, los hallazgos fueron de $40 \%$, mientras que, al aumentar el número de muestras a ocho, este porcentaje se duplicó a $80 \%$. Un aumento en el número de muestras únicamente fue significativo para hallazgos patológicos en mujeres mayores de 40 años. Sin embargo, en la práctica diaria esto puede consumir tiempo y recursos y existe el riesgo de sobrediagnóstico y sobretratamiento. Por su parte Cook y los otros ${ }^{18}$ demostraron la importancia y la necesidad de realizar un estudio microscópico de los especímenes de MR, ya que en su estudio se realizaron diagnósticos importantes en el $2.1 \%$ de los especímenes sin lesiones macroscópicas evidentes. Esto indica que se pueden presentar errores diagnósticos importantes con la toma de muestras insuficientes. Los especímenes generalmente son muy grandes, y la selección de dos muestras al azar para el análisis microscópico significa que solamente una pequeña proporción del tejido total será analizado.

Esto es especialmente cierto en pacientes sometidas a mastoplastia de simetría con antecedente de cáncer de mama contralateral. Una evaluación macroscópica detallada es esencial para identificar las zonas con anormalidades microscópicas, sin embargo, a menos de que la totalidad del tejido sea analizado microscópicamente, es imposible tener una certeza absoluta de la ausencia de lesiones. En nuestro centro, los especímenes de mastoplastia reductiva rutinariamente se valoran macroscópicamente y posteriormente se toman dos muestras al azar para su análisis microscópico. Si el estudio macroscópico revela alguna anormalidad, se toma un mayor número de muestras para el análisis microscópico. En este estudio, de 314 muestras patológicas, no se encontró ningún caso de patología maligna, incluso en pacientes con antecedente de cáncer de mama. Se encontró patología mamaria benigna en 43.3\% de las muestras analizadas, porcentaje inferior al encontrado en la literatura $(76.2 \%)^{2}$

En la mayoría de los estudios, la diferencia principal en el resultado de la incidencia de lesiones en muestras de MR parece ser el número de muestras patológicas que se analizan en el Departamento de Patología. 1,3,5,18-21 Dada nuestra muestra y la evidencia que existe en la literatura en cuanto al muestreo de la pieza patológica, la recomendación del autor es estandarizar el número de muestras analizadas microscópicamente con el fin de aumentar la sensibilidad del resultado final.

\section{CONCLUSIONES}

Las lesiones mamarias no siempre son evidentes en los estudios preoperatorios, por lo tanto, es indispensable realizar un estudio histológico de los especímenes de mastoplastia reductiva para aumentar la probabilidad de encontrar lesiones malignas o premalignas tras MR. Por lo tanto, concluimos que es válido y prudente preguntarnos ¿estamos procesando un número adecuado de muestras en pacientes de alto riesgo?

Sugerimos la realización de guías para la unificación de criterios en el análisis histopatológico de los especímenes obtenidos en MR y, una vez estandarizados, aplicarlos en nuestro propio centro.

Conflicto de intereses y financiamiento: Los autores declaran no tener ningún conflicto de intereses y no haber recibido patrocinio para la realización del trabajo. 


\section{REFERENCIAS}

1. Kececi Y, Tasli FA, Yagcı A, Sır E, Canpolat S, Vardar E. Histopathologic findings in breast reduction specimens. J Plast Surg Hand Surg. 2014; 48 (2): 122-125.

2. Tadler M, Vlastos G, Pelte MF, Tille JC, Bouchardy C, Usel M et al. Breast lesions in reduction mammaplasty specimens: a histopathological pattern in 534 patients. Br J Cancer. 2014; 110 (3): 788-791.

3. Ambaye AB, MacLennan SE, Goodwin AJ, Suppan T, Naud S, Weaver DL. Carcinoma and atypical hyperplasia in reduction mammaplasty: increased sampling leads to increased detection. A prospective study. Plast Reconstr Surg. 2009; 124 (5): 1386-1392.

4. Merkkola-von Schantz PA, Jahkola TA, Krogerus LA, Hukkinen KS, Kauhanen SM. Should we routinely analyze reduction mammaplasty specimens? J Plast Reconstr Aesthet Surg. 2017; 70 (2): 196-202.

5. Clark CJ, Whang S, Paige KT. Incidence of precancerous lesions in breast reduction tissue: a pathologic review of 562 consecutive patients. Plast Reconstr Surg. 2009; 124 (4): 1033-1039.

6. Carlson GW. The management of breast cancer detected by reduction mammaplasty. Clin Plast Surg. 2016; 43 (2): 341-347.

7. Petit JY, Rietjens M, Contesso G, Bertin F, Gilles R. Contralateral mastoplasty for breast reconstruction: a good opportunity for glandular exploration and occult carcinomas diagnosis. Ann Surg Oncol. 1997; 4 (6): 511-515.

8. Boice JD Jr, Friis S, McLaughlin JK, Mellemkjaer L, Blot WJ, Fraumeni JF Jr et al. Cancer following breast reduction surgery in Denmark. Cancer Causes Control. 1997; 8 (2): 253-258.

9. Baasch M, Nielsen SF, Engholm G, Lund K. Breast cancer incidence subsequent to surgical reduction of the female breast. $\mathrm{Br} /$ Cancer. 1996; 73 (7): 961-963.

10. Brown MH, Weinberg M, Chong N, Levine R, Holowaty E. A cohort study of breast cancer risk in breast reduction patients. Plast Reconstr Surg. 1999; 103 (6): 1674-1681.

11. Dupont WD, Page DL. Risk factors for breast cancer in women with proliferative breast disease. N Engl J Med. 1985; 312 (3): 146-151.
12. Hartmann LC, Degnim AC, Santen RJ, Dupont WD, Ghosh K. Atypical hyperplasia of the breast--risk assessment and management options. N Engl J Med. 2015; 372 (1): 78-89.

13. Desouki MM, Li Z, Hameed O, Fadare O, Zhao C. Incidental atypical proliferative lesions in reduction mammoplasty specimens: analysis of 2498 cases from 2 tertiary women's health centers. Hum Pathol. 2013; 44 (9): 1877-1881.

14. Colwell AS, Kukreja J, Breuing KH, Lester S, Orgill DP. Occult breast carcinoma in reduction mammaplasty specimens: 14-year experience. Plast Reconstr Surg. 2004; 113 (7): 1984-1988.

15. Dotto J, Kluk M, Geramizadeh B, Tavassoli FA. Frequency of clinically occult intraepithelial and invasive neoplasia in reduction mammoplasty specimens: a study of 516 cases. Int J Surg Pathol. 2008; 16 (1): 25-30.

16. Slezak S, Bluebond-Langner R. Occult carcinoma in 866 reduction mammaplasties: preserving the choice of lumpectomy. Plast Reconstr Surg. 2011; 127 (2): 525-530.

17 Aytac B, Sahsine T, Erturk FY, Kahveci R, Gokgoz S. Evaluation of incidence and histolopathological findings of breast lesions in reduction mammoplasty specimens: Uludag University experience. J Pak Med Assoc. 2013; 63 (7): 878-881.

18. Cook IS, Fuller CE. Does histopathological examination of breast reduction specimens affect patient management and clinical follow up? J Clin Pathol. 2004; 57 (3): 286-289.

19. Pitanguy I, Torres E, Salgado F, Pires Viana GA. Breast pathology and reduction mammaplasty. Plast Reconstr Surg. 2005; 115 (3): 729-734; discussion 735.

20. Ishag MT, Bashinsky DY, Beliaeva IV, Niemann TH, Marsh WL Jr. Pathologic findings in reduction mammaplasty specimens. Am J Clin Pathol. 2003; 120 (3): 377-380.

21. Blansfield JA, Kukora JS, Goldhahn RT Jr, Buinewicz BR. Suspicious findings in reduction mammaplasty specimens: review of 182 consecutive patients. Ann Plast Surg. 2004; 52 (2): 126-130.

22. Nielsen M, Thomsen JL, Primdahl S, Dyreborg U, Andersen JA. Breast cancer and atypia among young and middle-aged women: a study of 110 medicolegal autopsies. Br J Cancer. 1987; 56 (6): 814-819.

23. Bondeson L, Linell F, Ringberg A. Breast reductions: what to do with all the tissue specimens? Histopathology. 1985; 9 (3): 281-285. 